Conference abstract LPM02

\title{
Alternatives to Laboratory Animals: In Vitro and In Silico Approaches
}

\author{
S. Cascone ${ }^{1}$, F. De Santis ${ }^{1}$, G. Lamberti ${ }^{1}$, G. Titomanlio ${ }^{1}$, A. A. Barba ${ }^{2}$ \\ ${ }^{1}$ Dipartimento di Ingegneria Chimica e Alimentare, Università di Salerno, Fisciano, Italia \\ 2 Dipartimento di Scienze Farmaceutiche, Università di Salerno, Fisciano, Italia \\ E-mails: scascone@unisa.it (S. Cascone), fedesantis@unisa.it (F. De Santis), glamberti@unisa.it \\ (G. Lamberti), gtitomanlio@unisa.it (G. Titomanlio), aabarba@unisa.it (A. A. Barba)
}

Sci Pharm. 2010; 78: 589

doi:10.3797/scipharm.cespt.8.LPM02

The pharmaceutical industry uses million of animals for in-vivo studies to discover or to develop novel pharmaceutics. Time and costs involved and the extrapolation to human physiology of the data obtained are the main difficulties of these studies. The alternative to laboratory animals is the development of invitro and in-silico models (which are physical and mathematical models respectively).

In literature, different in-vitro models are proposed to reproduce the gastrointestinal tract taking in account both the mixing and the biochemical features. However, these models are not able to reproduce closely the real physiology. To describe the drug release in the human body, different kinds of mathematical models (in-silico models) are proposed. Among these, physiologically-based pharmacokinetic models are more complex and complete: the body is divided into compartiments, corresponding to real structures of the body, each with a specific function. Despite their reliability, these models require the fitting of a lot of parameters.

The aims of this work are to realize an in-vitro model reproducing the mechanical and biochemical features of the gastrointestinal tract and to develope (and validate) a novel physiologically-based model incorporating the pharmacokinetic and physiological parameters.

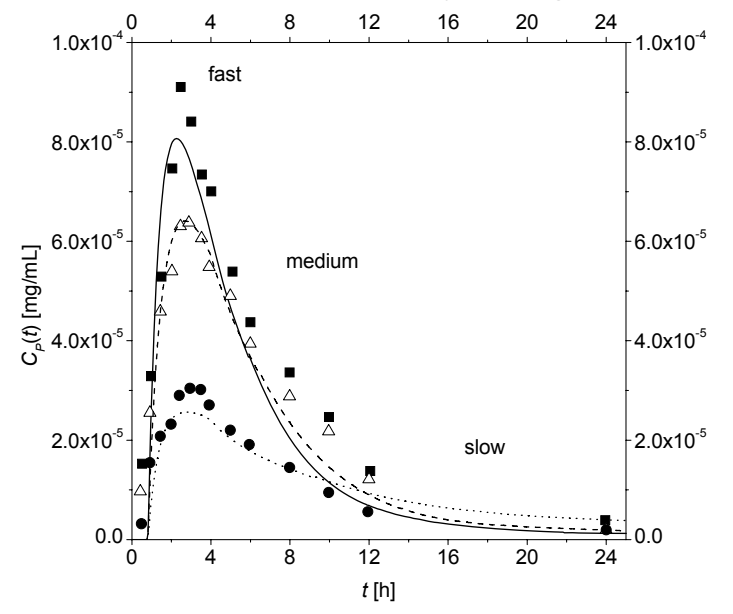

The proposed in-silico model is simple and is characterized by a limited number of parameters. Haematic drug levels after different kind of administrations have been successfully simulated.

Fig. 1. Haematic drug concentration after the administration from three solid dosage forms (fast, medium and slow release). Symbols: experimental data, curves: model prediction. 